\title{
Introduction
}

\author{
Managing Knowledge in Policymaking
}

C. W. WALI VAN LOHUIZEN

Eindhoven Technological University

MANFRED KOCHEN

University of Michigan

For many years questions concerning the utilization of research in policymaking and planning have focused on the mechanisms for the production of scientific knowledge. Yet problems of research utilization also require that we investigate the transfer mechanisms that bring research products to bear on processes of policymaking and planning. The complexity of these transfer mechanisms, together with differences in the character of scientific and political institutions, have often suggested to observers that it is useful to think in terms of $t$ wo cultures. But less attention has been paid to the utilization of knowledge that has not been packaged as an explicit product of scientific inquiry; to combining research findings with nonscientific knowledge; and to managing flows and stocks of knowledge in an architecture characterized by multiple sources and channels of communication.

This issue of Knowledge: Creation, Diffusion, Utilization presents the findings of a workshop held to shed light on these relatively new questions concerning knowledge utilization. All the contributions were first presented at the Workshop on Utilization-Focused Research and Planning: The Management of Knowledge in Policymaking. The

Authors' Note: We would like to express our deep appreciation to Charles Barr, a policy analyst currently at the University of Michigan, for his help in editing these articles.

Knowledge: Creation, Diffusion, Utilization, Vol. 8 No. 1, September 1986 3-11

(c) 1986 Sage Publications, Inc. 
workshop, held at Eindhoven Technological University, the Netherlands, in December 1984, was organized by Wali van Lohuizen. A forthcoming issue of the Journal of Architectural and Planning Research will include another set of papers by other workshop participants. The workshop's goal was to clarify and explain shifts in priority and emphasis in policy research, in policymaking, and in the analysis of the relationship between them.

The workshop met a need for a discussion of some of the issues and proposed approaches that concern policymakers, planners, and researchers. We wanted to bring the results to the attention of a wider community of persons concerned with the relation of knowledge to action in contexts of public policy. We are grateful to William Dunn for the opportunity to initiate communication with the readership of Knowledge, and for his editorial assistance.

\section{Shifts in Research}

Policy research has traditionally been conceived as scientific inquiry focused on a policy problem. That inquiry's purpose is to better conceptualize the problem or even to solve it. Some authors contend that policy research, because of the focus on utilization outside the sphere of scientific knowledge, differs from "pure" scientific inquiry in both its goals and its methodology. However, this perspective preserves the concept of policy research as a scientific activity primarily designed to produce knowledge.

Van Lohuizen argues that research and science institutions are characteristically faithful to a spirit of coping with data and information and relatively indifferent to the form of the research product. Science is traditionally presumed to create new knowledge. Dealing with information in a scientific way, however, does not necessarily result in "new knowledge": It might produce a better understanding of a problem still unsolved or a reorganization of knowledge structures. For example, a population projection designed to assess future housing needs must be redone if a new application to education planning is intended. Such processes of recapitulation, transformation, and translation call for restructuring and refocusing knowledge. An extension of these processes can be seen in the "engineering and advisory" model (van de Vall and Ulrich) of social science, in which research findings or new knowl- 
edge structures are linked to problem solutions. Scientific inquiry, in this model, produces not only knowledge and better understanding, but practical policy-specific applications.

Yet another source of problem solutions lies in the application of nonscientific knowledge (i.e., knowledge other than that resulting from research in scientific institutions). Experts and laymen in policy and planning institutions possess knowledge, insights, understanding, and judgments affecting how they conceptualize and manage the problems they are charged with solving. In policymaking, nonscientific knowledge should ideally be integrated with applicable research findings (Knott, van Lohuizen, Hart). Information system development that supports the successful orchestration of this blend is now under way.

Apart from the issue of absorbing research findings, the combined challenges of a shortage of high-quality information of whatever type and an overload of information flows from which to filter it threaten effective policy decision making (Leemans). Information technologies (MIS, DSS, AI, expert systems) provide leverage for meeting those challenges. Those technologies, systematized for the kinds of decisions policy actors make, provide valuable tools for streamlining and finetuning knowledge flows.

In summary, the research community is shifting from seeing research as scientific inquiry independent of policymaking to accepting that much research is becoming more integrated with policymaking, while retaining its scientific character.

\section{Shift on the Part of Policymaking}

It is not our goal, as guest editors of this special issue, to overview all recent progress in the disciplines supporting insights into policymaking. However, it is useful to offer a common framework for discussing the links between policy research and policymaking. We view relations between policymaking and policy research as falling into five types:

(1) Ignoring the findings of scientific inquiry as they may bear on policymaking.

(2) Research precedes and provides foundations for policymaking ("survey before you plan").

(3) Policymaking draws on research as an instrument and/or as an early warning mechanism. 
(4) Policymaking integrates research expertise mechanisms into its own system of procedures.

(5) Policies arise immediately from research, a position that is close to the mainstream of science-based comprehensive planning in the 1960 s and early 1970 s.

In a Type 3 relation research is considered both as an instrument and as a warning mechanism throughout the sequence of policymaking and planning stages. Research is used as an instrument providing data, information, knowledge, and insights/understanding whenever a need arises among policymakers. Another way of viewing this is to assume that the researcher plays a role within a policymaking team and performs research in this context alone. Yet performing the function of an early warning mechanism requires a critical attitude toward the deficiencies of policymaking processes and a readiness to complement policy goals and values with problem-specific, real-world knowledge.

In a Type 4 relation policy and research expertise is assumed to be mobilized in a single, doubly expert individual (Knott) who is capable of cognition and decisions that truly integrate research-specific and policyspecific knowledge and mechanisms.

We see planners shifting from either a Type 2 or Type 3 relation to Type 1 or Type 4, and from Type 5 to Type 1 or Type 3. Either scientific findings tend to be ignored in policymaking or policymaking tends to integrate research into its own procedures.

Other major shifts in the perception of the policy arena must be identified. One is the awareness that policymaking involves coping with multiactor situations, with no simplified "government/client service group" dichotomy possible. There are many stakeholders in any arena, and various divisions of government provide a part of the cast (Hart). The other shift is the increasing awareness that policymaking is a political activity - a contest over the distribution and application of power in a society-and that this also pertains to planning. The problems of mobilizing knowledge and policy in the planning professions per se are not a focus of this special issue, however.

In summary, policymakers tend to look at "research" (or, more broadly, at all providers of information and knowledge) as a set of utilitarian activities if they are left sufficient freed om of action and if the information and knowledge is specifically utilization-focused.

Now we turn to the issue of whether or not available knowledge and information will be used, provided policymakers require it. In addition, 
an important shift in thinking about policymaking will be illustrated. Policymaking is increasingly seen as a loose system of past policies that grow or change through the accretion of decisions over long periods of time, instead of as a planned architecture of choices, the nature and timing of which can be confidently forecast. This strengthens the choice of the first, third, and fourth types of relations between research and policy schematized in this section.

\section{Shifts in the Relations of Research and Policymaking}

In the argument of the previous section, the relations may fall, at the extreme ends of the spectrum, into two categories: (1) research and policymaking are different worlds, constituting two cultures, or (2) research and policymaking are two intimately interlocking systems of decisions and procedures for managing knowledge. The second category has no well-accepted nickname, but in this issue we refer to it as knowledge management or the knowledge household (van Lohuizen). The term household originates from a Germanic root that means keeping one's house in order, and it should be understood metaphorically. The two cultures concept does not apply for some types of relations, in terms of the type of utilization of knowledge presented: instrument use versus enlightenment (van de Vall and Ulrich). Instrument use represents a relatively tight relationship: Research is directed toward a well-defined utilization goal within a given policy-making process, and research findings are instrumental for problem solving within that process. Enlightenment takes place when knowledge from many different sources creeps into policymaking along ill-defined pathways, resulting in a general awareness of how things work or how they are structured. Policymaking as decision accretion strengthens the enlightenment function of research over the instrumental functions. It may pull researchers closer within the policymaking circle (Type 3 in the schema above) but it may also push the researchers farther outside those circles (Type 4)-the expert takes over-or to Type 1, in which research is ignored.

A closer relationship between researchers and policymakers will develop only if research adopts a new set of perspectives on the dynamics of research, of policymaking, and of their relations. This process has been described in Section 2 in terms of integrating research into pol- 
icymaking in ways reflecting trends in the handling and management of knowledge.

\section{Knowledge Management}

What are the processes involved in managing knowledge? We envision four functions:

(1) positioning knowledge in a policy process;

(2) extending "research" from mere production to the processing, storage, retrieval, dissemination, utilization, and general management of knowledge;

(3) facilitating and developing a philosophy and methodology of utilizationfocused research and of handling knowledge; and

(4) shifting "research" from producing certainty and complete knowledge to structuring ignorance and managing uncertainty.

As a concept, knowledge household may be useful for relating many relevant findings in the research utilization literature of the past 10 or 20 years. It may also be used as a device for structuring those findings. This is a research task yet to be accomplished.

As a tool, the knowledge management concept may serve both as a framework and as a structuring device within the dynamics of a policy process in order to manage data, information, knowledge proper, insight/understanding, and judgment, thus serving to inject intelligence into decision making. In this sense, it relates to central themes in each of the articles: Albinski's policy-oriented theory; van de Vall and Ulrich's advisory role of policy research and its optional professional paradigm; van der Heijden's decision support system for retail planning; Knott's roles of a professional in public policymaking; Leemans's case study of information advantage and monopoly and fragmented "households"; Kochen and Barr's distributed expert systems for more effective management of planning projects; and Hart's intermediary to mediate among conflicting perspectives and to integrate different forms and levels of knowledge.

Both as a concept and as a tool, knowledge household provides a framework for linking research and policy, stressing the positive roles of institutions dedicated primarily to one or the other activity. Managing knowledge is a researcher's task, in which mechanisms for responsibility 
and accountability differ radically from those confronting a policy decision maker. In policy arenas, decisions are volitional, actionoriented, and value-centered. In research, knowledge management is objective and reflective, prompted by the character of scientific inquiry.

Van de Vall and Ulrich's position on this point is different. They seem to gradually merge the role of the researcher (the sociologist) into the decision-maker role through the assumption of advisory functions. Knott more or less agrees, stressing the value of the expert who integrates research and policy talents. On the other hand, some authors stress the need for a different perspective: Leemans shows how limited an actor's scope is, particularly when large interests are at stake, and how barriers may be broken down by multiple interpretations of the problem at hand. Kochen and Barr propose to expedite the sharing of such interpretations within and among actor groups by mobilizing information technologies.

Knowledge management concepts facilitate integrating knowledge at every stage of a sequence of policymaking processes. They do so by encompassing a spectrum of knowledge types (causal, expert, scientific); by systematizing dissemination, transfer, and utilization; and by allowing for translation between regions on the spectrum from data to information to knowledge to insight/ understanding to judgment. Hart and van der Heijden offer illustrations of how this might be attempted. Hart, like Leemans, stresses the multiactor model, offering a solution to tackling problems by creating common ground with the help of knowledge management. Van der Heijden links knowledge management with the proven tool of decision support systems. This may open new avenues. Kochen and Barr offer another avenue of operationalizing the knowledge household by creating networks among experts, using so-called expert systems to create intelligent tools for handling knowledge.

As a concept and tool in policy processes, the knowledge household may further facilitate the development of a philesophy and methodology of utilization-focused research. The so-called proto-scientific character can serve as a starting point. Van de Vall and Ulrich's call for a professional paradigm is another point of departure, and Hart and van der Heijden provide pragmatic operating guidelines.

This host of concepts can help reinterpret research as an intervention in relation to uncertainty (i.e., not knowing the unexpected, as well as not knowing what to do) and ignorance (i.e, not knowing what is happening or what will happen). Research as applied to the manage- 
ment of knowledge elements in policy processes could be developed in either of two directions: the management of uncertainty rather than aiming at removing certainty, and provision of a structure of ignorance rather than the provision of all necessary and sufficient problem-specific knowledge. Managing uncertainty, without aiming at its elimination, is what is needed to strengthen the role of research. This constitutes a challenge. This special issue of Knowledge has tried to meet this challenge on a conceptual level to evoke further discussion and research.

\section{Structure and Contents of This Issue}

The issue opens with an article by van Lohuizen presenting the framework for all contributions. It spells out some of the shifts discussed in this introduction, exploring new concepts through which a better structuring of the field may be brought about. The hoped for result will be a deeper insight into the functional relations whose management calls for applying the central concepts of knowledge management and the knowledge household.

Leemans then presents a case study on information and decision making in a large-scale water project in the Netherlands. It illustrates many of the issues outlined by van Lohuizen and develops the idea of stakeholder information advantages. Saxe also describes cases of uses of social science knowledge by policymakers, and abstracts some general principles from these case studies (psychotherapy, alcoholism, polygraph testing). He shows how the U.S. Congress uses knowledge for its educative functions: It does not change decisions, but makes the discussion of public policy more reasoned, enlightened, and principled. He shows how policy research involves synthesis of ideas and data as well as the use of multiple perspectives.

Kochen and Barr further develop the concept of knowledge utilization developing a planning aid expert system by suggesting a role for $\mathrm{AI}$ and knowledge management system tools. This fits into the current need to interrelate tools and expertise belonging to both the private and public sectors.

Hart reports practical work on intermediary processes between actors and between "knowledge" and "policy," in which tools for managing knowledge have been both developed and tested. Multiactor situations are faced, nonscientific knowledge is handled, and commu- 
nication proceeds with the nurturing of mechanisms for shared organizational learning. Van der Heijden discusses the possible role of decision support systems in the field of urban planning for retail facilities. He highlights the need for a different way of performance research for planning applications: tailored more closely to decision-making stages while retaining distinct responsibilities special to research.

Knott analyzes the roles of the expert in public policymaking: neutral versus partisan, information-gathering versus goal-focused, immediate time frame versus long-term assessment. He explores differences between academics and policymakers. Both are experts, yet they differ in the knowledge bases constituting expertise, the ways in which they arrive at their decisions, and the goals that their decisions support. This theme is then related to the shifts in perspectives discussed above in this introduction.

Albinski argues that policy research will only see the optimization of its results when each research project makes reference to explicitly policy-oriented theory as well. He thereby draws a parallel with scientific inquiry and theory formation. Van de Vall and Ulrich hypothesize a relation between research and policy with an advisory model of sociological practice. In this model, research findings are merged with policy advice, blurring the boundaries between researchers, consultants, and policymakers.

\section{Conclusion}

From the previous statements on the shifts in perspectives that are occurring, relation Types 1,3 , and 4 are being strengthened whereas Types 2 and 5 are weakening. There is scope for the application of research products, the priorities of science may be jeopardized as some of its essential characteristics conflict with the priorities of policymaking. This creative tension is the problem addressed in this issue of Knowledge: Creation, Diffusion, Utilization. 